\title{
超快激光制造智能可调谐仿生复眼
}

\author{
马卓晨 ${ }^{1,2}$, 张永来 ${ }^{1}$, 孙洪波 ${ }^{1,2^{*}}$
}

1. 吉林大学电子科学与工程学院, 集成光电子学国家重点实验室, 长春 130012;

2. 清华大学精密仪器系, 精密测试技术及仪器国家重点实验室, 北京 100084

* 联系人, E-mail: hbsun@tsinghua.edu.cn

\section{Ultrafast laser fabrication of smart tunable bioinspired compound eyes}

\author{
Zhuo-Chen $\mathrm{Ma}^{1,2}$, Yong-Lai Zhang ${ }^{1} \&$ Hong-Bo Sun ${ }^{1,2^{*}}$ \\ ${ }^{1}$ State Key Laboratory of Integrated Optoelectronics, College of Electronic Science and Engineering, Jilin University, Changchun 130012, China; \\ ${ }^{2}$ State Key Laboratory of Precision Measurement Technology and Instruments, Department of Precision Instrument, Tsinghua University, Beijing 100084, \\ China \\ * Corresponding author, E-mail: hbsun@tsinghua.edu.cn
}

doi: 10.1360/TB-2020-0756

经过几千年的自然进化, 节肢动物的复眼已经形成一 种独特的视觉系统, 具有视野广、景深大、成像失真小、 对移动物体敏感等一系列独特的优点 ${ }^{[1 \sim 5]}$. 昆虫复眼通常 由分布在半球曲面(主眼)上的数百个紧密排列(100\%填充 因子)的小眼组成. 正是由于这种精致的结构, 昆虫能够以 高信噪比接收人射光, 并以大视场角 (高达 $360^{\circ}$ )从周围环 境中获取光信号. 近年来, 复眼的优良光学特性激发了人 们研究人工制造仿生复眼结构的极大兴趣, 并将其应用于 机器人视觉、医学内窥镜、监视设备和军事侦察等领域 ${ }^{[6 ~ 10]}$. 然而, 与人眼相比, 天然的复眼结构尽管有很长的景深, 但不同距离的物体却不能很好地分辨. 天然复眼都是由具 有固定焦距的小眼组成, 所以无法进行可变焦距成像. 因 此, 复眼在变焦距调谐成像中的应用受到限制. 如果能够 在有效利用复眼大视场角的前提下, 同时赋予其可调谐变 焦成像的能力, 则该类新型复眼结构将在宽视野可调谐成 像光学系统中发挥重要作用. 然而, 复眼通常具有复杂的 三维曲面结构, 制备难度较大, 其材质通常是玻璃、聚合 物等惰性透明材料. 因此, 对复眼每个小眼的变焦调谐一 直鲜有报道. 本研究团队 ${ }^{[10]}$ 采用飞秒激光双光子加工技术 制作了基于智能响应材料牛血清白蛋白(BSA)的可调谐复 眼结构, 成功解决了上述问题, 相关结果发表在Advanced Functional Materials.

昆虫复眼有许多紧密排列在一个大曲面上的小眼. 这 些位于不同位置的小眼能够探测来自不同方向的目标, 并 且获得的光信号通过后面的晶雉传输到被感光细胞包围 的感杆束. 因此, 许多节肢动物, 如昆虫和甲壳类动物, 可以达到较宽的视场. 相反, 脊椎动物 (如人类) 的眼睛通
常是单透镜系统, 它们的视野通常相对较小. 然而, 人眼 有变焦距成像的能力, 因为人眼内部的晶状体的形状可以 改变, 以此来调整焦距. 因此, 脊椎动物的眼睛可以聚焦 在不同距离的物体上, 在视网膜上形成清晰的图像. 为了 结合这两种眼睛的优点, 我们采用飞秒激光双光子直写技 术制作了一种智能复眼, 其材质为 $\mathrm{pH}$ 敏感的 BSA, 它具 有精细的三维形貌和可调焦的小眼(图 1). 由于 $\mathrm{pH}$ 变化引 起结构的膨胀和收缩, 从而复眼的视场角和焦距都可以动 态调节以获得清晰的图像. $\mathrm{pH}$ 调节 BSA 发生形变的机制 是由于 BSA 分子链存在羧基和氨基. 这些基团根据 $\mathrm{pH}$ 的 不同，可以发生去质子化或质子化现象，从而带不同的电 性. 因此这些基团之间的静电相互作用导致 BSA 结构的膨 胀或收缩, 从而引起复眼视场角和焦距的变化.

该工作利用飞秒激光直写制备了 BSA 一体的复眼结 构, 由于 BSA 在不同酸碱度条件下的膨胀和收缩效应, 整 个复眼发生形变. 由于 BSA 的等电点约为 $\mathrm{pH}$, 因此在 $\mathrm{pH} 5$ 的环境中, 复眼呈收缩状态, 此时具有最小的视场角. 而当 $\mathrm{pH}$ 偏离等电点时, BSA 会发生膨胀, 带来视场角的变 化. 与此同时，小眼的膨胀也会导致焦距的调节. 该研究 同时获得了可调的视野(视场角在 $35^{\circ} \sim 80^{\circ}$ 范围可调)和可 变的小眼焦距. 此外, 该工作除了对全 BSA 蛋白质复眼进 行直接制作外, 还成功展示了将智能 BSA复眼与传统光学 器件 (该工作以 SU-8 微透镜为例) 灵活集成以形成 BSA/SU-8 复合型复眼的能力. 其中, SU-8 为惰性材料, 因 此在 $\mathrm{pH}$ 变化的时候可以保持形貌不变, 也就是视场角的 稳定. 而 BSA 构成的小眼可以通过 $\mathrm{pH}$ 来调节焦距. 该类 复眼在固定视场下实现了近 $400 \%$ 的焦距调谐. 我们相信 


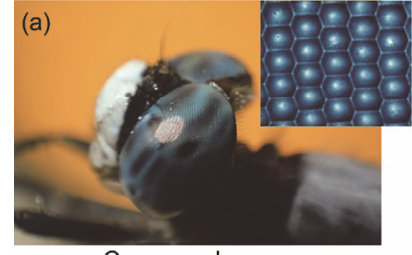

Compound eyes

(c)

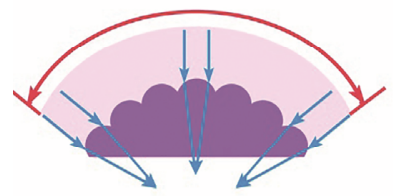

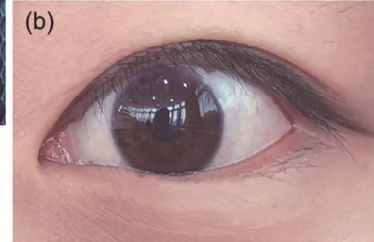

Human eyes

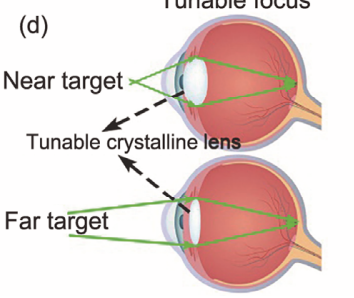

(e)

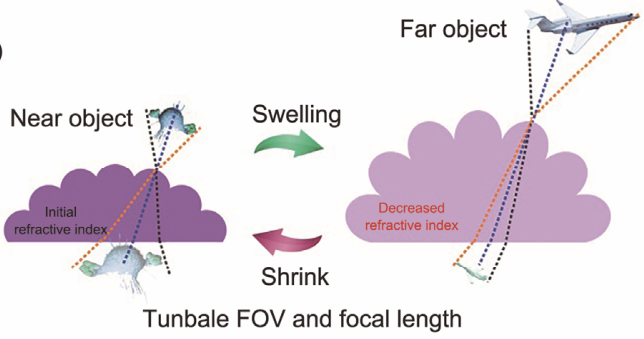

(f)
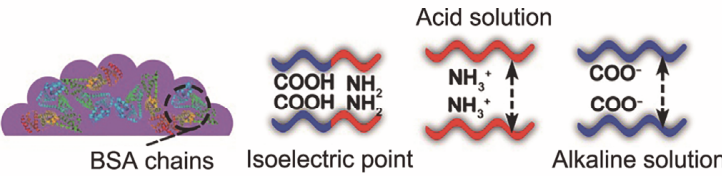

图 1 (网络版彩色) 可调谐蛋白质复眼示意图 ${ }^{[10]}$. (a) 蜻蜓复眼照片; (b) 人眼照片; (c) 复眼宽视野特性; (d) 人眼由可调晶状体实现的 变焦成像能力; (e) 蛋白质复眼可逆性膨胀和收缩特性的示意图, 实现可调节视野(FOV)和焦距; (f) 可调谐蛋白质复眼的基本机制

Figure 1 (Color online) A schematic of the tunable protein-based compound eyes ${ }^{[10]}$. (a) A photograph of compound eyes in dragonflies. (b) Photograph of human eyes. (c) Demonstration of the wide field of view of compound eyes. (d) Demonstration of the varifocal capability of human eyes with a tunable crystalline lens. (e) Schematic demonstration of the reversible swelling and shrinking characteristics of protein-based compound eyes that enable the tuning of the FOV and focal length. (f) Underlying mechanism of the tunable protein-based compound eyes

飞秒激光精密加工和集成基于智能响应材料的复眼结构 可能会为制造微型可调谐成像系统做出有益的探索, 在未 来光学成像智能微型系统的发展中具有重大的应用前景.

该工作利用 $\mathrm{pH}$ 响应型 BSA 蛋白的飞秒激光双光子 加工技术, 同时实现了兼具昆虫复眼(宽视场)和人眼(变 焦能力)优点的智能人工复眼的制作. 利用 BSA 蛋白的 $\mathrm{pH}$ 响应性膨胀和收缩效应, 通过改变周围溶液的 $\mathrm{pH}$ 成功实 现了视场和焦距的动态调节. 对于 BSA 一体的复眼结构, 视场角可从 $35^{\circ}$ 调整到 $80^{\circ}$. 除了 $\mathrm{BSA}$ 一体复眼的直接制 作外, 该工作还展示了 BSA 小眼与已有的微光学元件的 集成. 将 7 个紧密排列的小眼与 SU-8 微透镜组合成复合
复眼, 在改变 $\mathrm{pH}$ 时该复眼视场角可以保持稳定不变, 而 只调节小眼的焦距 $(400 \%$ 的调节范围). 飞秒激光双光子 加工基于蛋白质的智能复眼可以与传统的光学或光电设 备灵活集成, 展示了微型和智能成像系统的巨大潜力. 这 种可调谐复眼为可调谐成像提供了一个新的视角, 显示 出巨大的应用潜力, 如微型机器人的机器人视觉、大视场 监测的光流控设备、医学内窥镜诊断和微尺度粒子图像测 速, 其中可调谐成像和大视角检测是其突出的优势. 此 外, 考虑到 BSA蛋白良好的生物相容性, 智能复眼在生物 组织监测、活体成像和光流控芯片等方面可能会有广泛的 应用。

\section{推荐阅读文献}

1 Ko H C, Stoykovich M P, Song J, et al. A hemispherical electronic eye camera based on compressible silicon optoelectronics. Nature, 2008, 454: 748-753

2 Jeong K H, Kim J, Lee L P. Biologically inspired artificial compound eyes. Science, 2006, 312: 557-561

3 Lee L P, Szema R. Inspirations from biological optics for advanced photonic systems. Science, 2005, 310: 1148-1150

4 Song Y M, Xie Y, Malyarchuk V, et al. Digital cameras with designs inspired by the arthropod eye. Nature, 2013, 497: 95-99

5 Lee G J, Choi C, Kim D H, et al. Bioinspired artificial eyes: Optic components, digital cameras, and visual prostheses. Adv Funct Mater, 2017, 28: 1705202

6 Li J, Wang W, Mei X, et al. Artificial compound eyes prepared by a combination of air-assisted deformation, modified laser swelling, and controlled crystal growth. ACS Nano, 2019, 13: 114-124

7 Cheng Y, Cao J, Zhang Y, et al. Review of state-of-the-art artificial compound eye imaging systems. Bioinspir Biomim, 2019, 14: 031002

8 Keum D, Jang K W, Jeon D S, et al. Xenos peckii vision inspires an ultrathin digital camera. Light Sci Appl, 2018, 7: 80

9 Deng Z F, Chen F, Yang Q, et al. Dragonfly-eye-inspired artificial compound eyes with sophisticated imaging. Adv Funct Mater, 2016, 26: $1995-2001$

10 Liu X Q, Yang S N, Yu L, et al. Rapid engraving of artificial compound eyes from curved sapphire substrate. Adv Funct Mater, 2019, 29: 1900037 